\title{
MODULATING THE WETTABILITY CHARACTERISTICS AND BIOACTIVITY OF POLYMERIC MATERIALS USING LASER SURFACE TREATMENT
}

\author{
Paper \#M501 \\ D.G. Waugh, J. Lawrence, P. Shukla \\ Laser Engineering and Manufacturing Research Group, Department of Mechanical Engineering, \\ University of Chester, Parkgate Road, Chester CH1 4BJ, UK
}

\begin{abstract}
It has been thoroughly demonstrated previously that lasers hold the ability to modulate surface properties of materials with the result being utilization of such lasers in both research and industry. What is more, these laser surface treatments have been shown to affect the adhesion characteristics and bio-functionality of those materials. This paper details the use of a Synrad $\mathrm{CO}_{2}$ laser marking system to surface treat nylon 6,6 and polytetrafluoroethylene (PTFE). The laser-modified surfaces were analyzed using 3D surface profilometry to ascertain an increase in surface roughness when compared to the as-received samples. The wettability characteristics were determined using the sessile drop method and showed variations in contact angle for both the nylon 6,6 and PTFE. For the PTFE it was shown that the laser surface treatment gave rise to a more hydrophobic surface with contact angles of up to $150^{\circ}$ being achieved. For the nylon 6,6, it was observed that the contact angle was modulated approximately $\pm 10^{\circ}$ for different samples which could be attributed to a likely mixed state wetting regime. The effects of the laser surface treatment on osteoblast cell and stem cell growth is discussed showing an overall enhancement of biomimetic properties, especially for the nylon 6,6. This work investigates the potential governing parameters which drives the wettability/adhesion characteristics and bioactivity of the laser surface treated polymeric materials.
\end{abstract}

Keywords: $\mathrm{CO} 2$ laser; surface roughness; wettability characteristics; contact angle; polymers; bioactivity, stem cells, osteoblast cells.

\section{Introduction}

When two materials interact, the scientific term "wetting" is a fundamental phenomenon that can be taken into account when predicting how two materials will adhere to one another. The understanding of wetting of a surface by a liquid, leading to the spreading of those liquids over the surface, can be seen as a crucial factor that is adopted within surface chemistry and surface engineering including applications such as biomaterials [1-3] and coating technologies [4, 5]. As demonstrated in current surface engineering literature, wettability characteristics of many materials can be altered by the means of laser-induced surface treatment $[1,6,7]$ and other surface treatments [8-10]. Despite increased academic recognition and demand for greater industrial deployment, wetting and the effects of surface modification are still not fully understood within the engineering community. As a result, there is greater need of predicting wettability characteristics post laser processing since such work not only provides evidence for feasibility studies but also quantitatively evaluates the effectiveness of surface treatment.

Laser surface treatment offers numerous advantages such as it can be accurate, precise and non-contact allowing one to see that this can be a relatively clean process. In addition, with small heat affected zones lasers allow one to have the ability to modify both the surface chemistry and topography simultaneously without changing the bulk properties which may already be sufficient for the intended application. Furthermore, lasers also offer the opportunity of inducing varying levels of topography depending on how the laser is employed. For instance, periodic patterns can be induced using a focused beam whereas a more random pattern can be employed using a larger, more divergent, laser beam. Specific to polymers, infra-red lasers give rise to resonant coupling in the form of bond and lattice vibrations allowing for the processing to be thermolytical. This is due to the fact that the photon is only weakly absorbed by the polymer, with the energy that has been absorbed being distributed to vibrational modes [11]. This leads to melting and re-solidification of the material as the laser passes over the polymeric sample.

This work provides an overview of $\mathrm{CO}_{2}$ laser surface treatment of nylon 6,6 and polytetrafluoroethylene (PTFE) and discusses the its effects on the surface properties, wettability characteristics and bioactive nature with respect to mesenchymal stem cells and osteoblast cells. 


\section{Experimental Technique}

\section{$\mathrm{CO}_{2}$ Laser Surface Treatment}

A $\mathrm{CO}_{2}$ laser (60 W Firestar-ti; Synrad, Inc., USA) was employed at varying powers to irradiate the surfaces of nylon 6,6, and polytetrafluoroethylene (PTFE), which were initially mechanically cut in to samples of $10 \mathrm{~mm}$ x $10 \mathrm{~mm} \times 1 \mathrm{~mm}$. The laser was scanned across the surface of the samples using parallel line scans. The powers, scan speed and distance between the scanned lines for each sample are detailed in Table 1. Asreceived samples were also used to compare against the laser surface treated samples. The nylon as-received sample was designated the ID code NAR and the PTFE was designated the ID code PAR.

Table 1: Details of the powers, scan speeds and distance between scan lines for each sample.

\begin{tabular}{ccccc}
\hline $\begin{array}{c}\text { Sample } \\
\text { Type }\end{array}$ & $\begin{array}{c}\text { Sample } \\
\text { ID }\end{array}$ & $\begin{array}{c}\text { Power } \\
(\mathrm{W})\end{array}$ & $\begin{array}{c}\text { Scan } \\
\text { Speed } \\
\left(\mathrm{mms}^{-1}\right)\end{array}$ & $\begin{array}{c}\text { Distance } \\
\text { between } \\
\text { parallel } \\
\text { lines } \\
(\mu \mathrm{m})\end{array}$ \\
\hline $\begin{array}{c}\text { Nylon } \\
\text { 6,6 }\end{array}$ & N50_7 & 7 & 600 & 50 \\
Nylon & N100_7 & 7 & 600 & 100 \\
6,6 & & & & \\
Nylon & N100_10 & 10 & 600 & 100 \\
6,6 & P50_7 & 7 & 600 & 50 \\
PTFE & P100_7 & 7 & 600 & 100 \\
PTFE & P100_10 & 10 & 600 & 100 \\
PTFE & P10_ & & \\
\hline
\end{tabular}

\section{Topography and Surface Chemistry Analysis}

The surface profiles were determined using a white light interferometer (WLI) (NewView 500; Zygo, Ltd) with MetroPro and TalyMap Gold Software. The WLI was set-up using a $\times 10$ Mirau lens with a zoom of $\times 0.5$ and working distance of $7.6 \mathrm{~mm}$. This system also allowed the $\mathrm{Ra}$ roughness parameter to be determined for each sample.

All samples were analysed using $\mathrm{x}$-ray photoelectron spectroscopy (XPS). This allowed any surface modifications in terms of surface oxygen content due to the laser irradiation to be revealed. Details of the set-up and application of the XPS analysis can be found in [1].

\section{Wettability Characteristics Analysis}

The samples were ultrasonically cleaned in isoproponal (Fisher Scientific Ltd.) for 3 minutes at room temperature before using a sessile drop device to determine various wettability characteristics. This was to allow for a relatively clean surface prior to any contact angle $(\theta)$ measurements being taken. To ensure that the sample surfaces were dry a specimen dryer (Metaserv, Ltd.) was employed to blow ambient air across the samples. A sessile drop device (OCA20; Dataphysics Instruments, $\mathrm{GmbH}$ ) was used with relevant software (SCA20; Dataphysics Intrsuments, $\mathrm{GmbH})$ to allow the recent advancing $\theta$ for triply distilled water and the recent advancing angle for diiodomethane to be determined for each sample. Thereafter the advancing $\theta$ for the two liquids were used by the software to draw an OWRK plot to determine the surface energy of the samples. For the two reference liquids the SCA20 software used the Ström et al technique (triply distilled water - SFT(total:72.80), SFT(D:21.80), SFT(P:51.00); diiodomethane SFT(total:50.80), SFT(D:50.80), SFT(P:0.00)) to calculate the surface free energy of the material. It should be noted here that ten $\theta$, using two droplets in each instance, were recorded to achieve a mean $\theta$ for each liquid and surface.

\section{Bioactivity Analysis}

Prior to any biological testing being carried out, all samples were autoclaved (D-Series Bench-Top Autoclave; Systec, $\mathrm{GmbH}$ ) to ensure that all samples were sterilized. For all biological work undertaken, unless stated, a biological safety cabinet (BSC) (Microflow Class II ABS Cabinet; BioQuell UK, Ltd) was used to create a safe working environment and to provide a clean, sterile environment to manipulate the cells used.

Normal human osteoblast cells (Clonetics CC-2538; Lonza, Inc.) were initially cultured in a T75 $(75 \mathrm{ml})$ flask by suspending the cells in $19 \mathrm{ml}$ culture medium comprising of $90 \%$ eagle minimum essential medium (Sigma-Aldrich, Ltd., UK) and $10 \%$ foetal bovine serum (FBS) (Sigma-Aldrich Ltd., UK). To ensure the cells were ready for seeding they were re-suspended in $10 \mathrm{ml}$ of culture medium and dispensed between the samples in 6-well plates. This equated to $0.55 \mathrm{ml}$ $\left(2 \times 10^{4}\right.$ cells $\left./ \mathrm{ml}\right)$ for each sample. The well plates were then placed in the incubator for 24 hours (1 day) and 96 hours (4 days)

The Mesenchymal stem cells (Stem Cell Bank, Japan) were grown in tissue culture medium consisting of DMEM (with L-glutamine) (Sigma Aldrich, Ltd.), supplemented with $10 \%$ foetal calf serum (FCS) (Sigma Aldrich, Ltd.), and 100 units/ml of penicillin/and 0.1 $\mathrm{mg} / \mathrm{ml}$ of streptomycin (Sigma Aldrich, Ltd.), and placed in an incubator set at $37{ }^{\circ} \mathrm{C}, 5 \%$ humidified $\mathrm{CO} 2$ 
(Wolf Laboratories, Ltd.), throughout the study. When the cells reached subconfluence (70 to $80 \%$ ), they were retrieved with $0.25 \%$ trypsin and $0.02 \%$ EDTA. The retrieved cells were washed twice with PBS, centrifuged at $1200 \mathrm{rpm}$ for 12 minutes at room temperature and reseeded into 24-well plates cell culture at the initial seeding density of $5 \times 10^{4}$ cells per well. The well plates were then placed in the incubator for times between 24 hours ( 1 day) and 96 hours (4 days).

To analyse the affect on the bioactive nature of the laser surface treated samples the viable cells were counted following the 24 hours and 96 hours incubation time.

\section{Results and Discussion}

\section{Effects of Laser Surface Treatment on Topography}

As evidenced in Figure 1, the laser irradiated nylon 6,6 samples were considerably rougher in comparison to the as-received sample (NAR). The roughness for each of the laser surface treated nylon surfaces had increased and slight periodicity in the laser patterned samples was seen in contrast to the as-received sample (AR). In comparison to the as-received sample (AR), which had peak heights of the order of 0.1 to $0.2 \mu \mathrm{m}$, the peak heights for all of the laser treated samples were around $1 \mu \mathrm{m}$. It was also observed that the least periodicity arose from those samples which had patterns with 50 $\mu \mathrm{m}$ spacings. This can be seen to be of some importance as the laser spot size at the surface of the target samples was $95 \mu \mathrm{m}$ consequently allowing the scans for the trenches and hatch patterns to overlap and ultimately eliminate the natural periodicity of the original scanned pattern. However, the scan overlap occurring did ensure that the whole of the surface of the target sample was irradiated and modified in comparison to the nonirradiated reference sample (AR).

With regards to the PTFE (see Figure 2) it was observed that the surface roughness increased for samples P100_7 and P100_10 as a result of the $\mathrm{CO}_{2}$ laser surface treatment (see Table 2). This is in contrast to sample P50_7 which gave rise to a decrease in surface roughness. This is likely due to variations in the melting and solidification phenomenon and the large roughness which the as-received sample (PAR) had initially. That is, the $\mathrm{CO}_{2}$ laser, having a spot size of approximately 95 $\mu \mathrm{m}$ would have given rise to overlapped scanning during the laser scanning processing giving rise to melting of the surface, eradicating the initial roughness of the PAR sample during the re-solidification process.

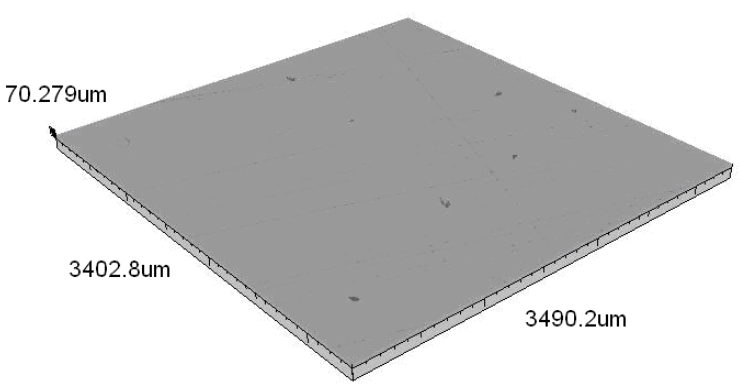

(a)

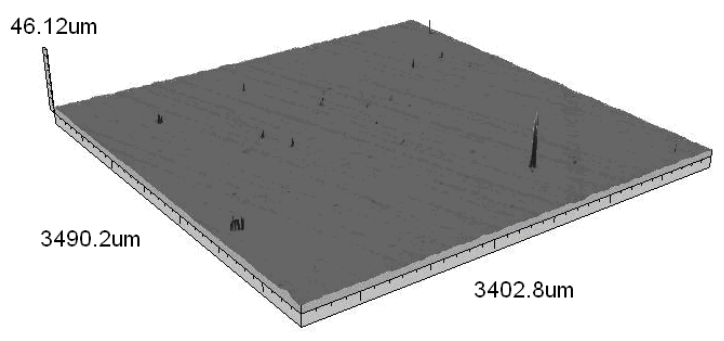

(b)

Figure 1: 3-D profiles of (a) as-received Nylon 6,6 (NAR) and (b) sample N100_7.

The results for surface roughness, contact angle and surface free energy, for each of the samples, are given in Table 2. As one can see, there were significant variations in the surface roughness when comparing the as-received samples with the corresponding laser surface roughened samples. The nylon 6,6 , following laser surface treatment, was rougher with an Ra of up to $0.83 \mu \mathrm{m}$ from a laser power of $7 \mathrm{~W}$; whereas a higher power of $10 \mathrm{~W}$ gave rise to a smoother surface with an $\mathrm{Ra}$ of $0.16 \mu \mathrm{m}$. This is likely due to the thermolytical nature of the $\mathrm{CO}_{2}$ laser-material interaction, bringing about a melting and solidification phenomenon similar to what was observed with the PTFE samples. 


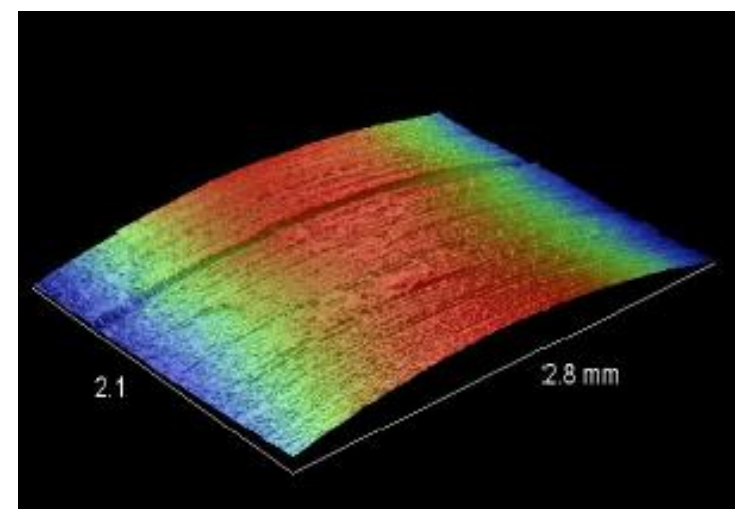

(a)

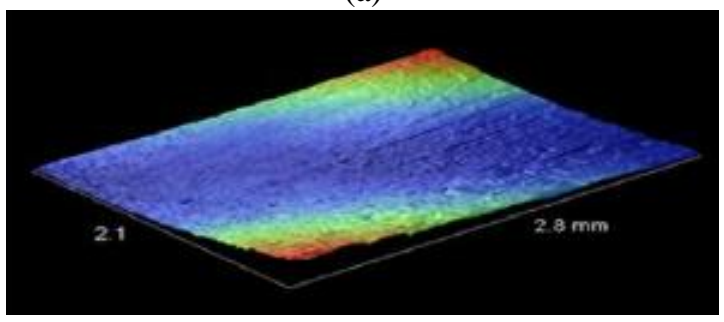

(b)

Figure 2: 3-D profiles of (a) the as-received PTFE and (b) sample P50_7.

\section{Effects of Laser Surface Treatment the Wettability Characteristics}

Table 2 also provides the data obtained for the wettability characteristics for each sample: the contact angle and the surface free. Similar to the surface roughness, the contact angle was significantly modulated as a result of the $\mathrm{CO}_{2}$ laser surface treatment. For the PTFE samples, the contact angle increased following laser surface roughening, when compared to the corresponding as-received samples, making them more hydrophobic with contact angles of up to $150^{\circ}$. The contact angle for nylon 6,6 decreased on account of the laser surface treatment and, along with previous work that has been carried out with nylon 6,6 , highlights a potential mixed state wetting regime resulting from an observed increase in surface roughness [1, 2]. This particular result shows that the interface between the liquid and surface is complex and other parameters such as surface chemistry and surface charge need to be accounted for across all of the samples before definite conclusions can be made.

The material variations from polymer to polymer is believed to be one of the main reasons as to the modulation in laser-material interaction and resulting wettability characteristics. Nylon 6,6, for instance, has a high strength, stiffness and temperature resistance but it does have higher water absorption and less chemical resistance. These properties are the result of the polymer chain structure as the nylon 6,6 has more amide linkages per chain meaning that it has more inter-chain bonding. These particular properties, especially the high water absorption, linked with the $\mathrm{CO}_{2}$ laser surface treatment has given rise to a more hydrophilic nylon 6,6 surface. Although, it should be noted that more research is required to ascertain the driving forces for this hypothesized mixed state wetting regime. In contrast, PTFE is a fluorocarbon, meaning that it only contains fluorine and carbon. The emergence of oxygen containing functional groups, arising from the melting and re-solidification process of the laser surface roughening, could have had a large impact upon the contact angle, enabling the PTFE to become more hydrophobic. With this in mind, it is important that further work be carried out relating to bond and functionalization analysis of the laser surface treated samples so that these hypotheses can be confirmed.

Table 2: Surface roughness, contact angle and surface free energy data for each sample.

\begin{tabular}{cccc}
\hline Sample ID & $\begin{array}{l}\text { Surface } \\
\text { Roughness, } \\
\text { Ra }(\mu \mathrm{m})\end{array}$ & $\begin{array}{l}\text { Contact } \\
\text { Angle }\left(^{\circ}\right)\end{array}$ & $\begin{array}{l}\text { Surface } \\
\text { Free } \\
\text { Energy } \\
\left(\mathrm{mJm}^{-2}\right)\end{array}$ \\
\hline NAR & 0.29 & $66.49 \pm$ & $43.88 \pm$ \\
& & 0.32 & 0.20 \\
N50_7 & 0.64 & $66.0 \pm 4.0$ & $40.87 \pm$ \\
& & $57.50 \pm$ & 2.39 \\
N100_7 & 0.83 & 2.50 & $1.94 \pm$ \\
N100_10 & 0.16 & $62.20 \pm$ & $44.00 \pm$ \\
& & 2.30 & 1.80 \\
PAR & 1.99 & $91.40 \pm$ & $28.35 \pm$ \\
& & 2.05 & 1.27 \\
P50_7 & 1.58 & $147.5 \pm$ & $1.58 \pm$ \\
& & 1.43 & 0.03 \\
P100_7 & \multirow{2}{*}{2.50} & $117.03 \pm$ & $13.15 \pm$ \\
& & 1.93 & 1.03 \\
P100_10 & \multirow{2}{*}{3.40} & $140.44 \pm$ & $1.48 \pm 0.06$ \\
& & 0.33 & \\
\hline
\end{tabular}

On account of laser surface treatment the surfaces of a number of polymeric materials can be modified with respect to their wetting and adhesive properties. As a result, laser surface treatment does hold the ability to modulate the adhesion characteristics of polymeric materials in order to enhance the surface properties for a number of applications involving adhesion.

\section{Effects of Laser Surface Treatment on Bioactivity}

It can be seen from Figure 3, for the nylon 6,6 samples, that the number of mesenchymal stem cells increased 
over time, especially in the presence of the laser surface treated nylon 6,6 samples. The manner of reaction seen in the cells themselves was variable depending on the processing parameters used. The 24 hour results were generally lower than those seen at the longer incubations. This is due to the fact that when cells are seeded onto a surface, the cells go through a lag phase before entering the growth phase. In the vast majority of samples all readings were higher than those found with sample NAR and sample PAR, as well as this, incubation over time also yielded higher readings. The lowest cell count $\left(10 \times 10^{3}\right)$ arose on the PAR sample following 24 hours of incubation and can be attributed to the fact that PTFE is inherently hydrophobic, having poor adhesion characteristics. This highlights that the laser surface treatment of the nylon 6,6 and PTFE samples significantly augmented the mesenchymal stem cell growth when compared to the as-received samples (NAR and PAR).

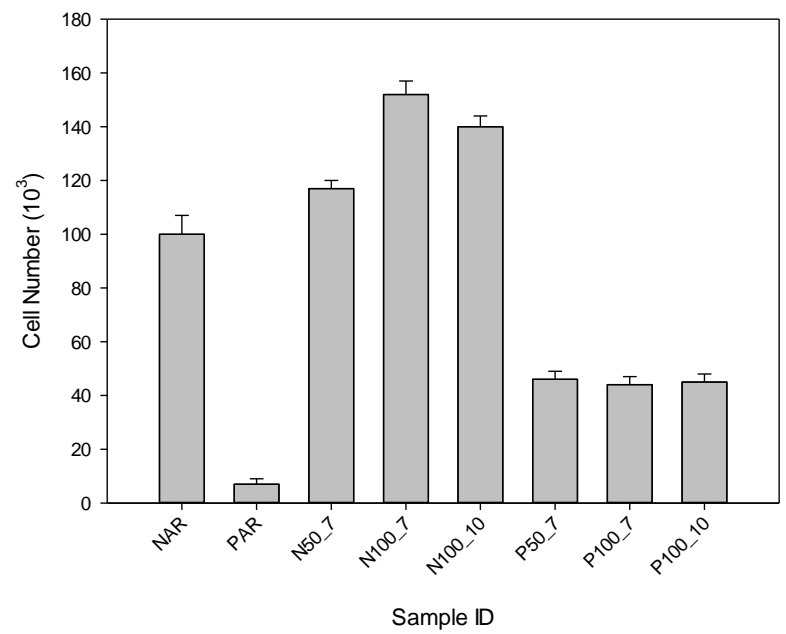

Figure 3: Stem cell count for each sample following 24hrs incubation.

A mesenchymal cell count after the 96hr (4 day) incubation period provided the opportunity to determine that the $\mathrm{CO}_{2}$ laser surface treated nylon 6,6 samples had given rise to a higher cell count with up to 250,000 cells/ml when compared to the as-received sample (NAR) and PTFE samples which had cell counts of approximately 78,000 cells $/ \mathrm{ml}$. This allows one to ascertain that even after 4 days incubation period with cover densities tending towards $100 \%$, the $\mathrm{CO} 2$ lasersurface treated nylon 6,6 samples still gave rise to enhanced cell response in terms of cell growth.

Following 24 hours and 96 hour incubation times, it was found that for both the nylon 6,6 and PTFE laser surface treated samples the stem cell number count had increased significantly compared to the respective as- received materials, NAR and PAR. This result highlights the potential of laser surface treatment of polymers for the enhancement of stem cell growth. What is more, viability tests showed that the number of viable stem cells on the laser surface treated samples, following the two incubation times, was higher when compared to the respective as-received materials.

From the materials characterization it was identified that there was an increase in surface roughness and an increase in surface oxygen content - both of which are known to have an impact upon the biomimetic properties of material surfaces [2,12-14]. These could be the main drivers which govern the stem cell reaction to the laser surface treated surfaces although research is still continuing to confirm this.

Following further experimental work with osteoblast cells, it was found that the cell count number on all laser surface treated samples gave rise to an increase in cell number. A similar trend to that of the mesenchymal stem cells. For example, Figure 4 shows that the osteoblast cell number increased following 96 hours incubation more when compared to the NAR sample. This was also seen with the PTFE samples although, like with the mesenchymal stem cells, the PTFE samples gave rise to a less significant increase in cell number and can be attributed to the hydrophobic nature of the PTFE samples.

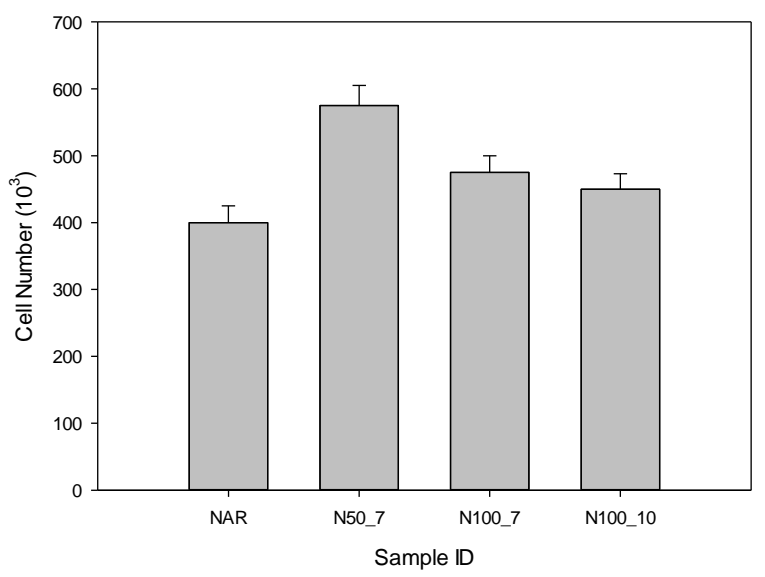

Figure 4: Osteoblast cell number for the Nylon 6,6 samples following 4 days of incubation.

\section{Conclusions}

For all $\mathrm{CO}_{2}$ laser surface treated nylon 6,6 and PTFE samples it was found that all samples gave rise to an increase in viable stem cell count number and viable osteoblast cell count number compared to the asreceived samples (NAR and PAR). It is believed that 
this is due to the increase in surface roughness, modulation of the adhesion characteristics and the increase in surface oxygen content of the samples; however, it has not yet been ascertained which is the main driving parameter governing the biomimetic nature of laser surface treated polymeric materials. That being said, in order for the biomimetic governing parameter to be identified more research is required.

As the importance of bioengineering increases with an aging population the significance and importance of manipulating biological environments will also increase. With this in mind, this work has shown the high feasibility of implementing a $\mathrm{CO}_{2}$ laser to surface treat two different polymeric materials so that the adhesion characteristics and bioactive nature of those materials can be enhanced.

\section{References}

[1] Waugh, D.G. \& Lawrence, J. (2011) Wettability and osteoblast cell response modulation through UV laser processing of nylon 6,6, Applied Surface Science 257 8798-8812.

[2] Waugh, D.G., Lawrence, J., Morgan, D.J. \& Thomas, C.L. (2009) Interaction of CO2 laser-modified nylon with osteoblast cells in relation to wettability, Material Science and Engineering C 29 2514-2524.

[3] Waugh, D.G. \& Lawrence, J. (2011) On the use of $\mathrm{CO} 2$ laser induced surface patterns to modify the wettability of poly(methyl methacrylate) (PMMA), Optics and Lasers in Engineering 48 707-715.

[4] Harnett, E.M., Alderman, J. \& Wood, T. (2007) The surface energy of various biomaterials coated with adhesion molecules used in cell culture, Colloids and Surfaces B 55 4970-4980.

[5] Zhao, Q., Wang, C. \& Liu, S. (2007) Bacterial adhesion on the metal-polymer composite coatings, International Journal of Adhesion and Adhesives 27 8591.

[6] Khosroshahi, M.E., Mahmoodi, M. \& Tavakoli, J. (2008) Effect of Nd:Yttrium-aluminium-garnet laser radiation on Ti6Al4V alloy properties for biomedical applications, Journal of Laser Applications 20 209-217. [7] Liang, F., Lehr, J., Danielczak, L., Leask, R. \& Kietzig, A.M. (2014) Robust non-wetting PTFE surfaces by femtosecond laser machining, International Journal of Molecular Sciences 15 13681-13696.

[8] Jung, Y.C. \& Bhushan, B. (2006) Contact angle, adhesion and friction properties of micro- and nanopatterned polymers for superhydrophobicity, Nanotechnology 17 4970-4980.

[9] Jung, Y.C. \& Bhushan, B. (2007) Wetting transition of water droplets on superhydrophobic patterned surfaces, Scripta Materialia 57 1057-1060.
[10] Zanini, S., Barni, R., Della Pergola, R. \& Riccardi, C. (2014) Modification of the PTFE wettability by oxygen plasma treatments: influence of the operating parameters and investigation of the ageing behaviour, Journal of Physics D: Applied Physics 47 1-9.

[11] Skordoulis, C.D., Makropoulou, M., \& Serafetinides, A.A. (1995) Ablation of nylon-6,6 with UV and IR lasers, Applied Surface Science 86 239-244. [12] Makropoulou, M., Serafetinides, A.A. \& Skordoulis, C.D. (1995) Ultra-violet and Infra-red Laser Ablation Studies of Biocompatible Polymers, Lasers in Medical Science 10 201-206.

[13] Hao, L. \& Lawrence. J. (2005) Laser Surface Treatment of Bio-Implant Materials. John Wiley \& Sons Inc.

[14] Chan, C.W., Hussain, I., Waugh, D.G., Lawrence, J. \& Man, H.C. (2012) In vitro mesenchymal stem cell responses on laser-welded NiTi alloy, Materials Science \& Engineering C 33 1344-1354.

\section{Meet the Author}

Dr David Waugh joined the University of Chester as Programme Leader for Mechanical Engineering in May 2014 and is a founding member of the Laser Engineering and Manufacturing Research Group (www.chesterlasers.org). Dr Waugh has published 2 books, 5 book chapters, 12 journal papers and 11 conference papers in laser material processing, healthcare engineering and wettability/adhesion characteristics. On account of Dr Waugh's wide ranging expertise he is a journal manuscript reviewer for 13 international journals including Materials Science and Engineering C, Proceedings of the IMechE Part C and Surface and Coatings Technology. 